\title{
Correction to: DDAH-1, via regulation of ADMA levels, protects against ischemia-induced blood-brain barrier leakage
}

\author{
Yichen Zhao $\cdot$ Xiaoye Ma $\cdot$ Yuchen Zhou $\cdot$ Junchao Xie $\cdot$ Xueyuan Liu $\cdot$ Yanxin Zhao (D)
}

Published online: 2 August 2021

(c) The Author(s), under exclusive licence to United States and Canadian Academy of Pathology 2021

\section{Correction to: Laboratory Investigation \\ https://doi.org/10.1038/s41374-021-00541-5}

Following publication of the original article, the authors have noted two errors in Figs. 6-7.
1. In Fig. 6B, the TUNEL staining image of SHAM group was wrongly used. The correct Fig. 6 is provided below.

2. In Fig. 7A, the TTC staining image of 3-day/KO group was incorrect. Figure 7 should appear as shown below.

All authors apologize for the errors and agree with the author correction. The original article has been corrected. 

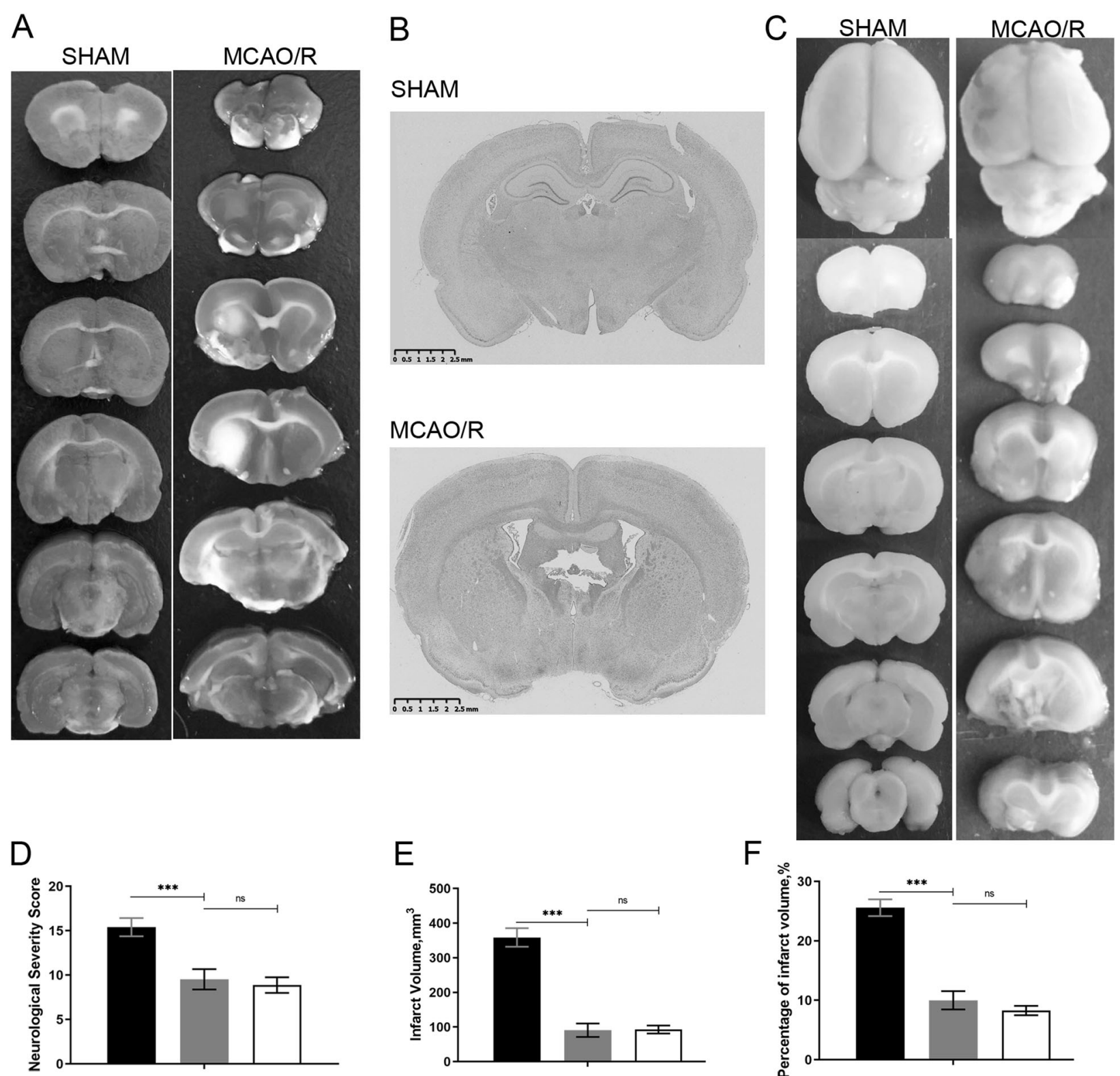

E

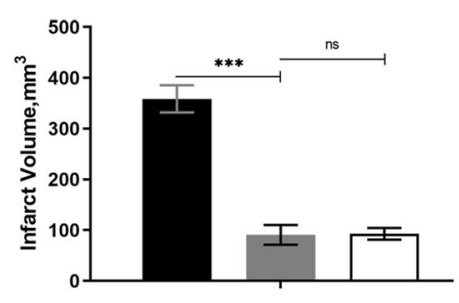

$\mathrm{H}$

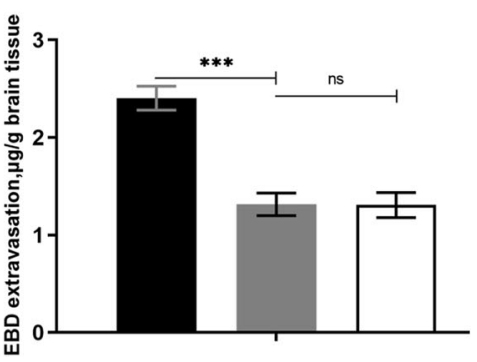

F

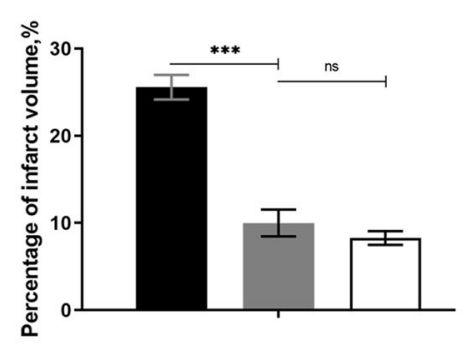

- $\mathrm{KO}$

ARG

$\square$ WT

Fig. 6 

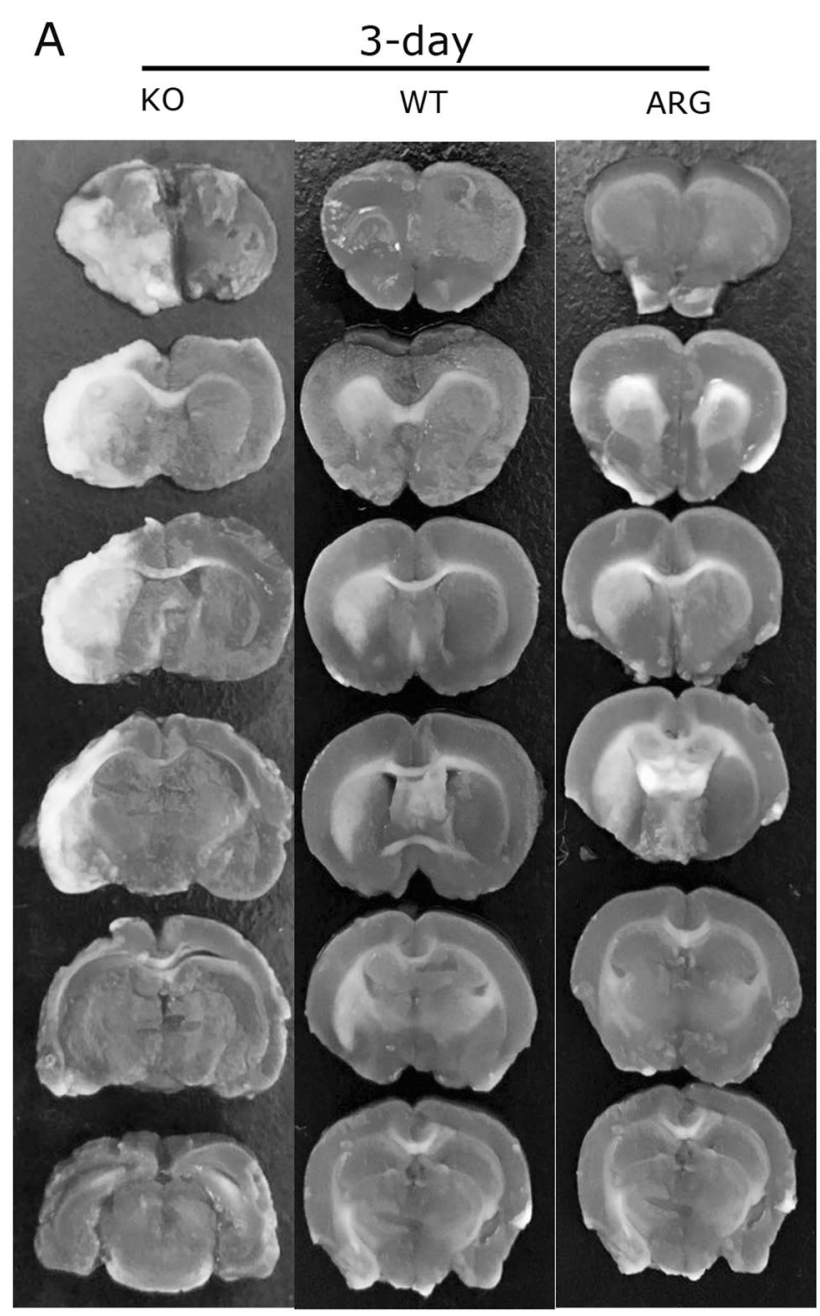

C

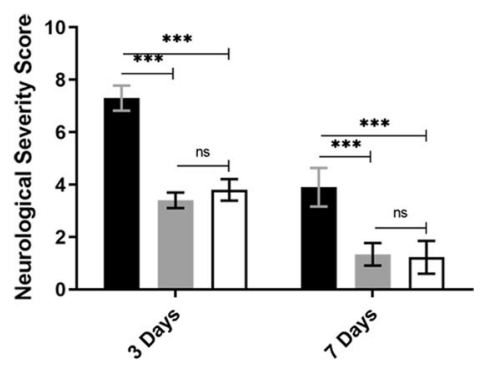

B

$\frac{7-d a y}{\text { KO WT }}$

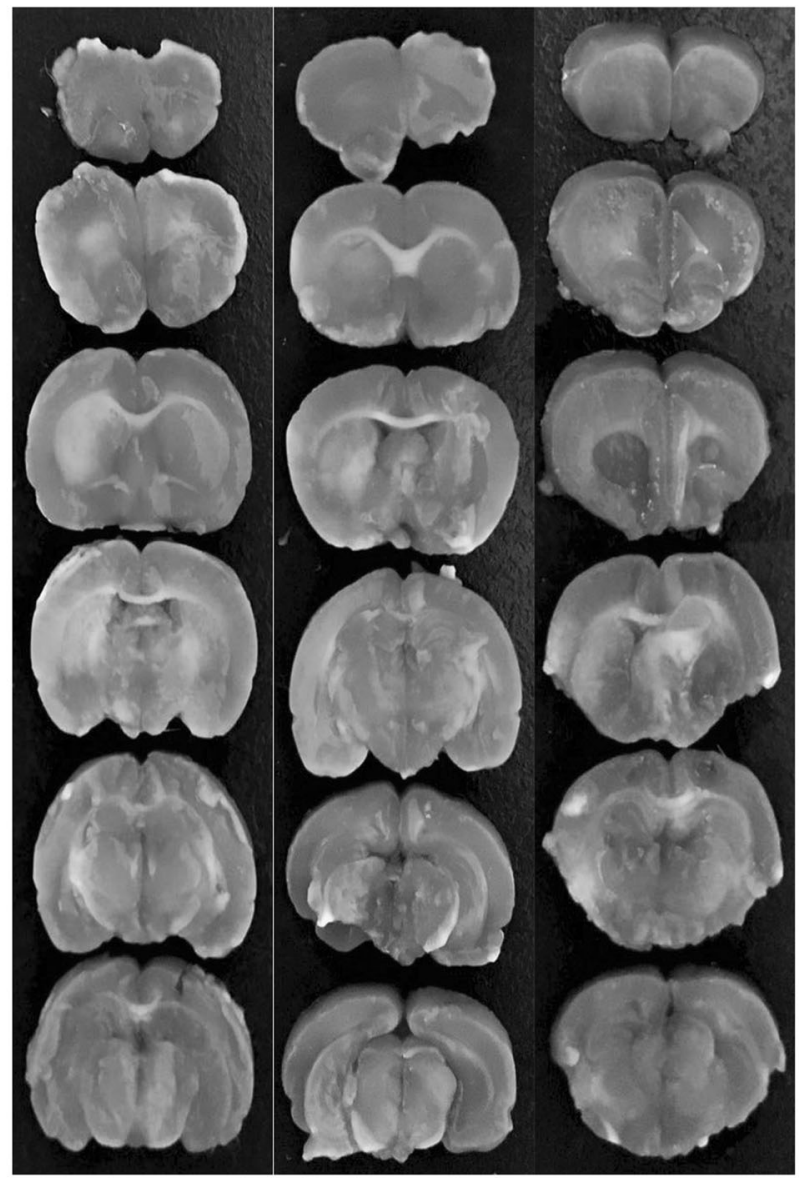

E

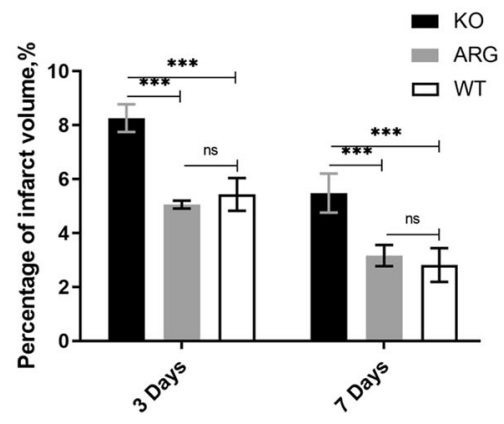

Fig. 7 